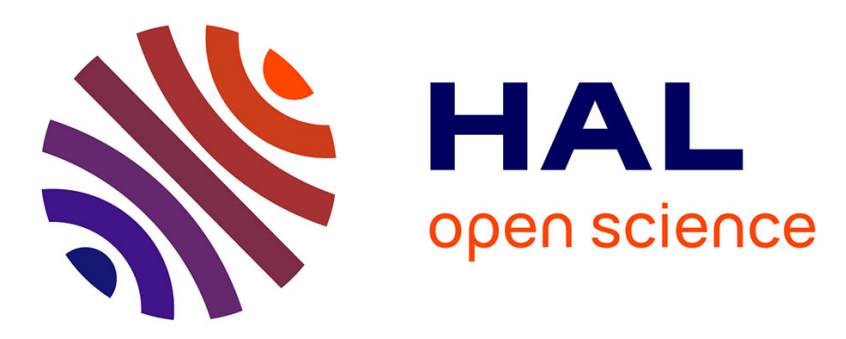

\title{
Evaluation Precision of a new Hybrid Indoor Localization System
}

Adel Thaljaoui, Nejah Nasri, Thierry Val, Samir Mahfoudi, Damien Brulin

\section{To cite this version:}

Adel Thaljaoui, Nejah Nasri, Thierry Val, Samir Mahfoudi, Damien Brulin. Evaluation Precision of a new Hybrid Indoor Localization System. 15th International Wireless Communications and Mobile Computing Conference (IWCMC 2019), Jun 2019, Tanger, Morocco. 10.1109/IWCMC.2019.8766626 . hal-02178644

\section{HAL Id: hal-02178644 https://hal.laas.fr/hal-02178644}

Submitted on 30 Jun 2020

HAL is a multi-disciplinary open access archive for the deposit and dissemination of scientific research documents, whether they are published or not. The documents may come from teaching and research institutions in France or abroad, or from public or private research centers.
L'archive ouverte pluridisciplinaire HAL, est destinée au dépôt et à la diffusion de documents scientifiques de niveau recherche, publiés ou non, émanant des établissements d'enseignement et de recherche français ou étrangers, des laboratoires publics ou privés. 


\section{Evaluating Precision of a new Hybrid Indoor Localization System}

\author{
Adel THALJAOUI \\ University of Toulouse \\ UT2J, CNRS-IRIT-IRT \\ Toulouse, France \\ adel.thaljaoui@gmail.com
}

\author{
Nejah NASRI \\ University of Sfax \\ ENIS, ,LETI \\ Sfax, Tunisia \\ nejah.nasri@isecs.rnu.tn
}

\author{
Thierry VAL \\ University of Toulouse \\ UT2J, CNRS-IRIT-IRT \\ Toulouse, France \\ val@irit.fr
}

\author{
Sami MAHFOUDHI \\ Department of MIS, CBE \\ Qassim University \\ Buraidah, KSA \\ s.mahfoudhi@qu.edu.sa
}

\author{
Damien BRULIN \\ University of Toulouse \\ UT2J, LASS-CNRS-N2IS \\ Toulouse, France \\ damien.brulin@laas.fr
}

\begin{abstract}
Bluetooth Low Energy (BLE) has revealed for decades manyinteresting opportunities especially in the field of positioning that made of it one of the most used technologies in indoor localization solutions. The system we propose is a combination of BLE, Acoustic and LiFi technologies. Our approachis not just arithmetic but using real time computed metrics that act precision indicators or "Dilution of precision" (DOP) weights of the results given by each technology. Depending on theseDOP-likeindicators, we were able to judge how much precise and accurate the returned results are.Our new approach was confirmed during experiments showing that the precision of this new hybrid system is much better when using these new precision metrics.
\end{abstract}

Keywords-Indoor Localization;BLE; LiFi; Acoustic; precision; Accuracy, DOP

\section{INTRODUCTION}

Indoor localization has become a very important domain of experiments due to all the difficulties that GPS is facing while operating inside buildings. Therefore, considering more suitable technologies come to be more than important, especially when it is question of health care. Indeed, due to the growing number of old persons in the last decades [1], many worries about their quality of life and their personal security in their own homes came to surface especially when these people have to live alone. This was one of our objectives when studying indoor localization using new technologies.
When dealing with one of the localization systems in indoor places, there are many challenges caused by the complexity of these environments, such as multipath effects, noise and path loss that affect the accuracy of these technologies. Unfortunately, the causes of these negative effects cannot be all removed, but could be attenuated by adding some corrective metrics.

In our work, we propose a new hybrid indoor positioning system that combines three different technologies[2][3] in order to compute the coordinates of a target inside a room; Bluetooth Low Energy (BLE), Acoustic and $\mathrm{LiFi}$. In order to improve the performance of our hybrid indoor positioning system, we used three new "precision metrics": BLE_DOP, Acoustic_DOP and LiFi_DOP. The final estimated coordinates of our target will be in this form:

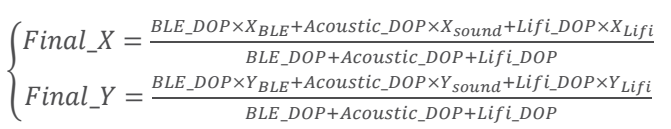

In this paper we present our work on improving precision and accuracy in an indoor localization system. In the following section, we present the main indoor localization techniques. Then we detail our proposed indoor positioning system and its components. In the fourth section, we present our approach of evaluating the precision and the accuracy of the results returned by this new hybrid system. Finally we present the main experiments and obtained results. 


\section{INDOOR LOCALIZATIONTECHNIQUES}

Indoor localization is a process enabling the determination of the position of a mobile target [7][8]. This process is generally achieved on two main steps [9]: ranging and positioning (Fig.1).

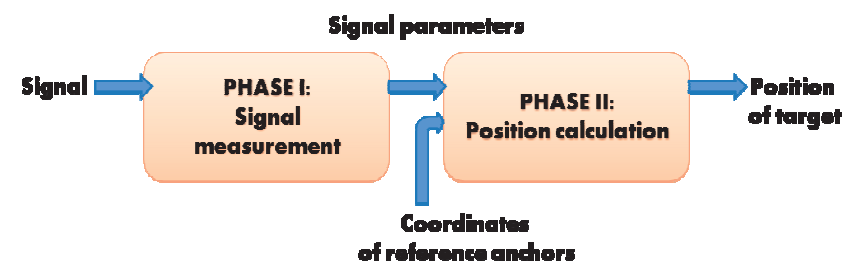

Fig. 1. Indoor Localization Process [9]

In the first step, signal proprieties are investigated using appropriate techniques in order to estimate the range of the signal, either distance or angle, such as ToA (Time of Arrival), TDOA (Time Difference of Arrival), DoA (Direction of Arrival) and RSS (Received Signal Strength).In the second step, the position of the target is computed using signal parameters and the coordinates of reference anchors. This step is based on many methods such as triangulation and trilateration.

\section{POPOSED HYBRID LOCALIZATION SYSTEM}

We propose a new indoor hybrid localization system that combines many positioning techniques and technologies. It is established around three sub-systems; BLE, Acoustic and $\mathrm{LiFi}$ as presented in the following figure.

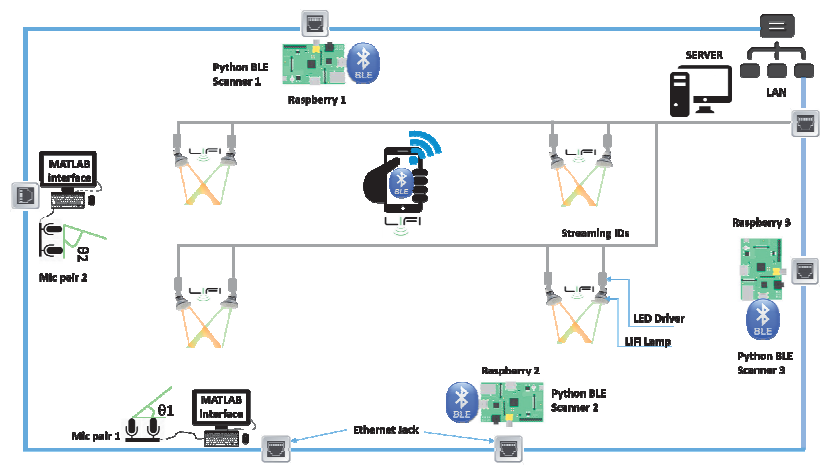

Fig. 2. Poposed Hybrid indoor localization system

In this paper, we detail our new "precise" and "accurate" indicators that we use to rate the precision of each sub-system(radio, audio or light): BLE_DOP, Acoustic_DOP and LiFi_DOP.

\section{A. BLE localization sub-system}

In our previous work on BLE indoor localization [10], we proposed a localization system based on Log-Distance Path Loss model and performing on threesteps as showed in Fig.3:

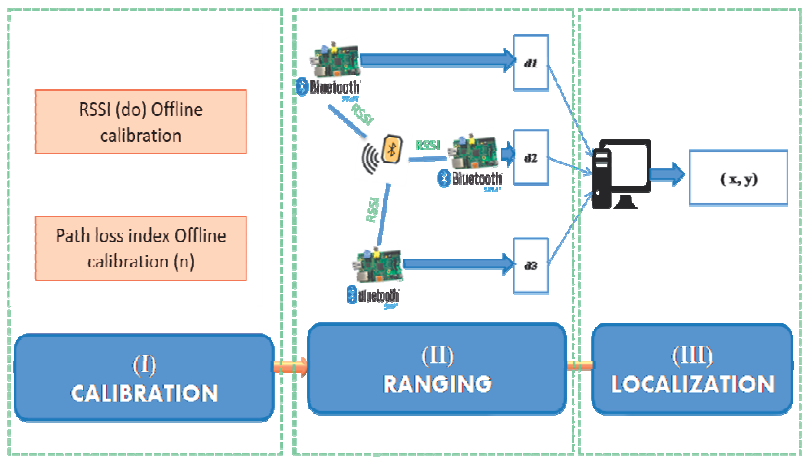

Fig. 3. Proposed BLE localization sub-system

- Phase 1: Calibration Phase

In this phase, we consider environment changes in our propagation model. As our system is composed of fixed beacons (with known coordinates) besides the mobile beacon, our idea is to exploit this information about known coordinates as well as inter-beacons distances in order to compute the path loss index for propagation model applied for these three beacons as well as $\operatorname{RSSI}\left(d_{0}\right)$. The frequency of this calibration is function of dynamic changes in the room.

For path loss index calibration, the idea of the offline training is to apply BLE propagation model for these three fixed model (two by two), compute the path loss index for every couple $\left(\mathrm{n}_{1-2}, \mathrm{n}_{2-1}, \mathrm{n}_{3-2}, \mathrm{n}_{2-3}, \mathrm{n}_{1-3}, \mathrm{n}_{3-1}\right)$ and finally calculate the mean of the above indexes:

$n \_$mean $=$mean $\left(v_{1-2}, v_{2-1}, v_{3-2}, v_{2-3}, v_{1-3}, v_{3-1}\right)$

For known distances, path loss index can be estimated as follows:

$$
n=\frac{R S S I\left(d_{0}\right)-R S S I(d)}{10 \times \log \left(\frac{d}{d_{0}}\right)}
$$

- $\quad$ Phase 2: Ranging Phase

As we already "know" the value of the path loss index, we estimate distance using following formula:

$$
d=10^{\frac{R S S I\left(d_{0}\right)-R S S I}{10 \times n}}
$$

- Phase 3: Position estimation

In this phase, the system estimates the position of the BLE receiver device using trilateration algorithm on its linear form:

$$
\left(\begin{array}{l}
x_{B L E} \\
y_{B L E}
\end{array}\right)=A^{-1} b
$$

Where:

$$
A=\left(\begin{array}{ll}
\left(x_{3}-x_{1}\right) & \left(y_{3}-y_{1}\right) \\
\left(x_{3}-x_{2}\right) & \left(y_{3}-y_{2}\right)
\end{array}\right) \text { and } b=\frac{1}{2}\left(\begin{array}{l}
d_{1}^{2}-d_{3}^{2}+D_{13}^{2} \\
d_{2}^{2}-d_{3}^{2}+D_{23}^{2}
\end{array}\right)
$$


$D_{13} \quad\left(D_{23}\right.$ respectively) is the distance between BLE beacon $B_{1}$ and BLE beacon $B_{3}$ ( BLE beacons $B_{2}$ and $B_{3}$ ).

B. Acoustic localization sub-system

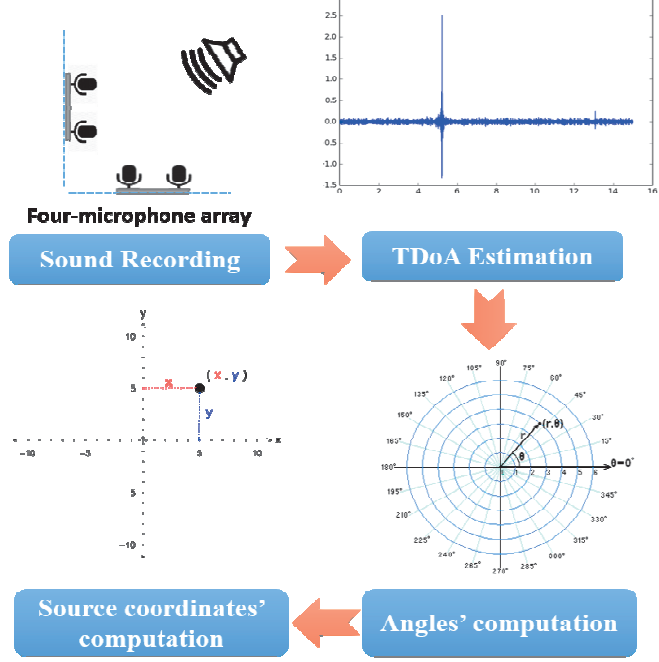

Fig. 4. Sound positioning sub-system [12]

In our previous work [3][12], we considered an acoustic source localization system composed of an array of two pairs of microphones recording simultaneously a sound (Fig.5.). Using this recorded sound, the system computes time difference between the microphones of each pair and then determinates the directions of arrival (angles $\theta_{12}$ and $\theta_{34}$ ) of the sound on them using cross-correlation.

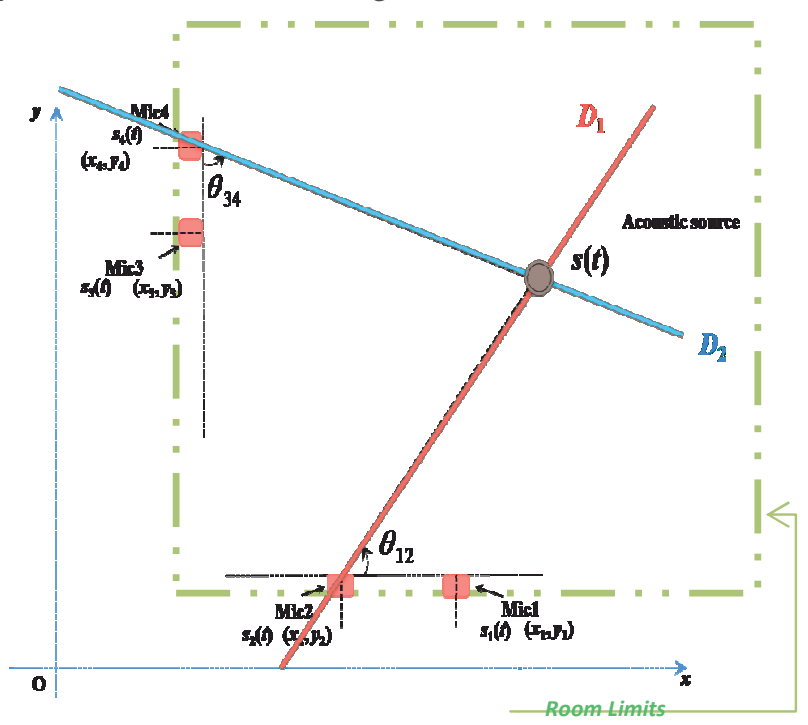

Fig. 5. Sound position estimation [12]

- Finally, after estimating angles $\theta_{12}$ and $\theta_{34}$ and assuming that sound waves are planar, equations of the two lines crossing the first (respectively the second) microphones pair and the acoustic source can be determined as follows:

$$
y=a_{12} x+b_{12}
$$

$$
y=a_{34} x+b_{34}
$$

where the coefficient $a_{12}, b_{12}, a_{34}$ and $b_{34}$ can be computedusing Triangulation. The coordinates of the acoustic source are deduced through the resolution of the equation of intersection of the two equations above.

\section{LiFi localization sub-system}

LiFi( Light Fidelity) was first used by Harald Haas in his TED Global talk on Visible Light Communication [15]. It is implemented using LED devices. Normally, these bulbs are used for rooms' illumination by applying a constant current. LEDs can be switched "ON" and "OFF" very quickly. Using these very quick variations, data can be encoded in the light in order to generate a new data stream by varying the flickering rate of the LED: if the LED is on, a digital 1 is transmitted, if the LED is off, the transmitted digital is 0 .

In our study case, we propose a LiFi sub-system made out of five pairs of LiFi LEDs deployed in the roof of the room as shown in Fig.below:

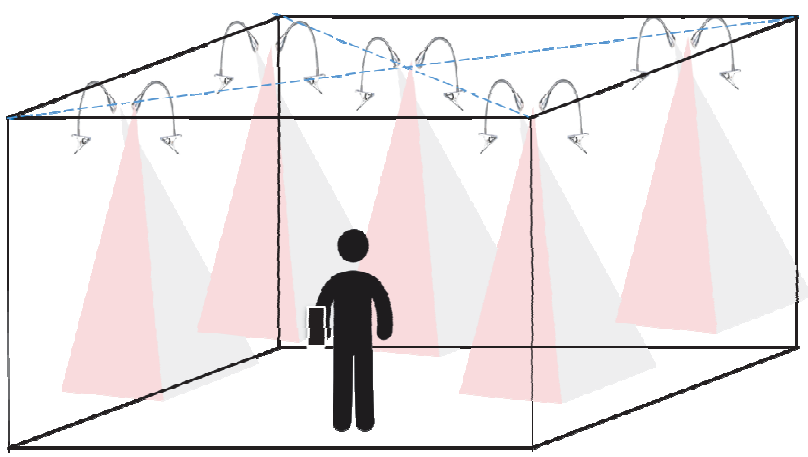

Fig. 6. LiFi positioning sub-system

Our approach is to use VLC technology enabled in every LiFi LED to transmit its ID to the photo detector in the LiFi dongle that we can imply connect to a smartphone. This component will convert the received light signal to an electrical one. Hence, if the smartphone detects the Li-Fi signal from the Li-Fi LED lamps, and identifies which lamp is transmitting data through its transmitted ID. For more precision, we use the crossing of two LiFi LED spectra as shown below:

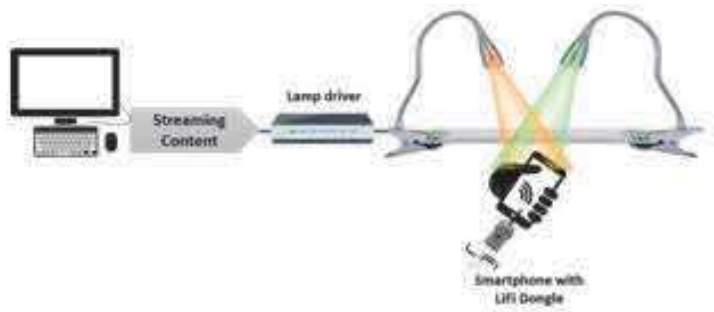

Fig. 7. LiFi localization

So, we can discern three cases:

- The smartphone does not detect any LiFi lamp. 
- The photo detector identifies the IDs of two LiFi lamps. The smartphone is located at the center of the crossing of the two LEDs'spectra.

- The smartphone only detect one LiFi lamp ID. It is located in the limit of the LED spectrum.

\section{ACCURACY AND PRECISION EVALUATION IN PROPOSED HYBRID INDOOR LOCALIZATION}

Each indoor positioning system works in a different range that goes from 5 meters to 50 meters depending on the type of the used technology [11]. In fact, for ultrasound and vision based positioning systems, coverage are between $1 \mathrm{~m}$ to $10 \mathrm{~m}$. However, even inside range of coverage of some technologies, estimated results can present errors that could lead to positioning system uncertainty. This issue of precision and accuracy is considered one of the most challenging subjects that needs to be investigated.

In positioning systems terminology, "accuracy" refers to the difference between the estimated location and the real one while "precision"refers to the repeatability of a particular computed position over many measurements [13].

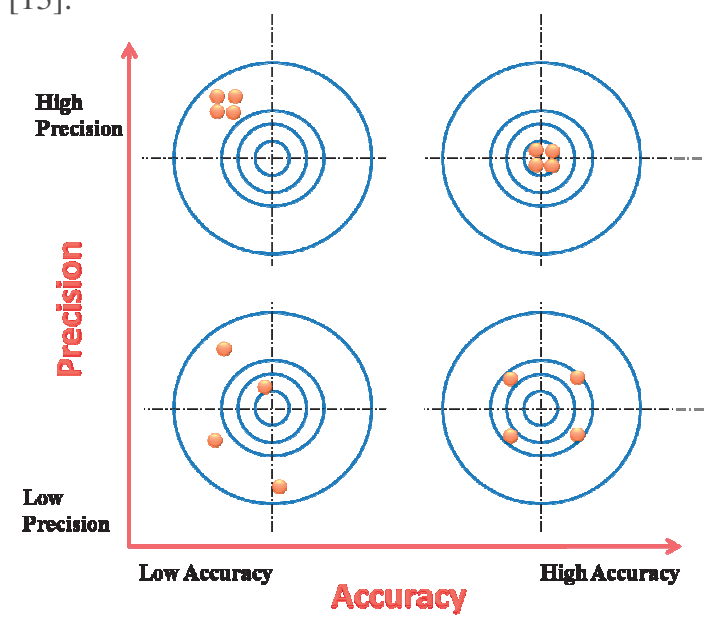

Fig. 8. Precision vs Accuracy [13]

With the advent of new indoor positioning systems, it has been urgent to select a metric that indicates how precise and accurate the estimated location is.

One of the oldest Precision metrics is GPS DOP (Dilution of Precision). In spite of its outdoor exclusivity, this metric has been an inspiration for many studies to propose an indoor "DOP-like" indicator based on the same assumptions. Some indoor positioning systems use another concept when evaluating their result: "confidence indicators".In our work we were inspired of these two concepts by adapting them to each technology and technique we used..

\section{A. BLE sub-system Precision Metrics}

RSSI is not a reliable measure [4][5]. It is very sensible to environment changes and presents consequently, high levels of noise, caused for example, caused by multi-path reflections. In fact, as we could see while studying this technology, the larger the distance between BLE receiver and BLE emitter, the lower the signal strength is.But,distance estimations contain a lot of noise whichwould lead to many errors. Precision inicator should take into account these facts and be able to show how much precise and accurate the returned results are.

Therefore, in our formulation of BLE precision indicator, we follow two main steps: outliers rejection using the "Modified Thompson $\tau$ method" [6] and BLE_DOP formulation. We propose the following algorithm:

\section{During RANGING PHASE:}

- Measuring N RSSI values for each beacon

- Outliers Removal using Thompson $\tau$ method

- Compute DOP_BLER

During POSITIONING PHASE:

- Outliers Removal using Thompson $\tau$ method

- Compute DOP BLEP

Finally: BLE_DOP $=($ DOP_BLER + DOP_BLEP $) / 2$

Where :

$$
D O P_{-} B L E R=\sum_{i=1}^{3} \frac{e^{-\left|\frac{R S S I_{i}}{R S S I_{\min }}\right|}}{3}
$$

and

$$
D O P_{-} B L E P=\frac{1}{G P S_{-} D O P_{-} \text {Like }}=\frac{1}{\sqrt{\text { trace }(Q)}}
$$

\section{B. Acoustic sub-system Precision Metrics}

In order to estimate the accuracy of the Acoustic subsystem, we use the notion of reproductivity of estimates for the same position. Thus, at the time of the location, during the ranging phase, the system performs $\mathrm{N}$ sound captures and then eliminates the outliers that could deviate our judgment on accuracy. This operation will be repeated during the phase of posiyioning. We propose the following algorithm:

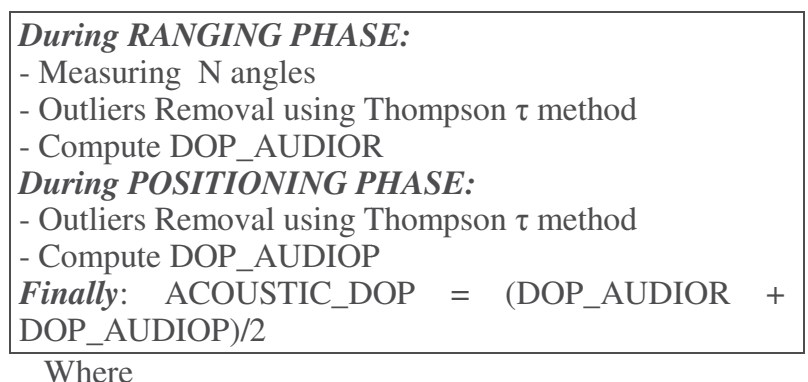

$$
D O P_{A U D I O R}(\theta i)=1-\frac{2 \times S T D(\theta i)}{\pi} ; i \in\{1,2\}
$$

and 


$$
D O P_{\text {AUDIOP }}=\frac{1}{G P S_{-} D O P_{-} \text {Like }}=\frac{1}{\sqrt{\operatorname{trace}(Q)}}
$$

\section{EXPERIMENTS}

Ourproposed hybrid indoor localizationsystem was validated by performingmany experimental tests on each sub-system apart and then on their combination. Therefore we implemented a prototype of a smart room composed of:

- Three computers (RAM more than 2Go), one of them as the server and the two others for acoustic localization

- Two bars in each we connected two electret microphones and a jack connection.

- Three raspberries with aBLE dongle each for BLE RSSI scan.

- These computers and raspberries are connected through a local network (LAN) using fixed IP addresses.

- A smartphone (Samsung S6 edge plus) in which we installedan android app for BLE signal emission

For audio localization validation, we developed a MATLAB code that uses sockets for network data transmission to a remote server.

Server MATLAB final interface, as given in yhe figure belowestimates DOP indicators and returns four locations:

- Sound position estimated by audio sub-system (black star point)

- Mobile position estimated by BLE sub-system (red star point)

- Hybrid position obtained by the combination of the two sub-systems results using DOP indicators (red point)

- $\quad$ Real position (black point)

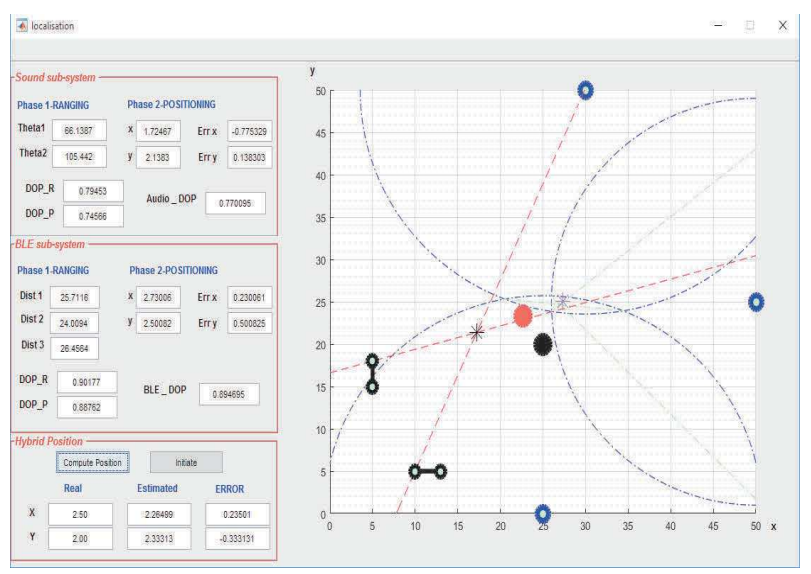

Fig. 9. MATLAB interface for hybrid location estimation

The final figure(Fig.10.) shows computed hybrid coordinates for the same real locations as well as corresponding errors:

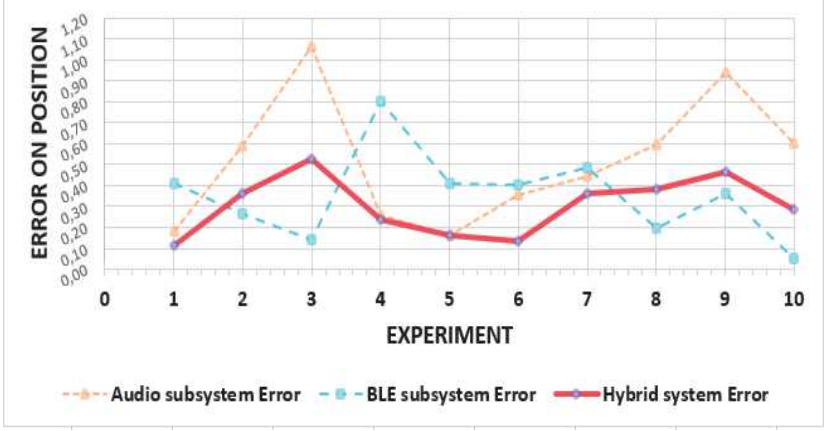

Fig. 10. Error minimization using proposed hybrid approach

\section{CONCLUSION AND FUTURE WORK}

In this paper, we discussed the different experiments we have carried out along the way in which we implement our hybrid approach to the three indoor localization technologies (audio, radio and LiFi).

At the end of these experiments, the results obtained allow us to conclude that our approach based on precision indicators acting as weights gives more precise results in the order of $0.3 \mathrm{~m}$ than every sub-system acting alone.

\section{REFERENCES}

[1] Eurostat. (2014, Mars) Population statistics at regional level. [Online].

http://epp.eurostat.ec.europa.eu/statistics_explained/index.php/Pop ulation_statistics_at_regional_level

[2] Adel Thaljaoui, Thierry Val, Damien Brulin and Nejeh Nasri, "Localisation d'une source sonore par un réseau de microphones", Journées Nationales des Communications Terrestres, JNCT 2014 Toulouse-Blagnac - France., 2014.

[3] Adel Thaljaoui, Thierry Val, Damien Brulin and Nejeh Nasri, "Real Time Acoustic Localization of Elderly Persons in a Smart Home," The International Conference on Performance Evaluation and Modeling in Wired and Wireless Networks (PEMWN2014), November 04 - 07, Sousse, Tunisia 2014.

[4] M. KAYTON and W.R. FRIED, Avionics Navigation Systems, 2nd ed., New York: Wiley, 1997, 773 pp

[5] R. THOMPSON. A Note on Restricted Maximum Likelihood Estimation with an Alternative Outlier Model. Journal of the Royal Statistical Society. Series B (Methodological), Vol. 47, No. 1 (1985), pp. 53-55

[6] John M. CIMBAL, Outliers, Penn State University, 2011

[7] M.Depsey, "Indoor Positioning Systems in healthcare", Radiance Inc. White paper, 2003

[8] El Khediri, S., Nasri, N., Wei, A., \& Kachouri, A. Probabilistic Energy Value for Clustering in Wireless Sensors Networks. Wireless Sensor Network, 5(2), 26, 2013

[9] Da Zhang, Feng Xia, Zhuo Yang, Lin Yao, "Localization Technologies for Indoor Human Tracking", 5th International Conference Future Information Technology (FutureTech), 2010

[10] Adel Thaljaoui, Thierry Val, Nejah Nasri, Damien Brulin. BLE Localization using RSSI Measurements and iRingLA (regular paper). Dans : IEEE International Conference on Industrial Technology (ICIT 2015), Seville, 17/03/15-19/03/15, IEEExplore digital library, (en ligne), mars 2015. 
[11] Luca Mainetti, Luigi Patrono, Ilaria Sergi,"A Survey on Indoor Positioning Systems", Department of Innovation Engineering, University of Salento, Lecce, ITALY, 2014

[12] Adel THALJAOUI, Thierry VAL, Damien BRULIN and Nejeh NASRI, "Localisation d'une source sonore par un réseau de microphones", Journées Nationales des Communications Terrestres, JNCT 2014 Toulouse-Blagnac - France., 2014.

[13] Retimana, E., Kealy, A. \& Hale, M. "Concepts of Position Repeatability and Position Reliability using the Global Positioning System", 2004

[14] Li-wei Chan, Ji-rung Chiang, Yi-chao Chen, Chia-nan Ke, Jane Hsu, Hao-hua Chu, "Collaborative Localization: Enhancing WiFiBased Position Estimation with Neighborhood Links in Clusters", Volume 3968 of the series Lecture Notes in Computer Science pp 50-66. 